\title{
The end of artefacts
}

Bill Phillips celebrates a beautiful reformation of the metric system, by which scientists measure the physical world, coming into effect on World Metrology Day, 20 May 2019.

T he Système Internationale d'Unités (SI) had its origins in the French revolution, whose egalitarian philosophy envisioned a system of units "For all times, for all people". In the greatest revolution for units since the French revolution, redefinitions of the kilogram, ampere, kelvin and mole will soon see all seven base units of the SI defined by fixing the values of constants of nature, finally doing away with the artefact standards that defined the metric system from its inception.

Originally, the metre was to be $10^{-7}$ of the Earth's equator-to-pole distance. After a careful partial survey ${ }^{1}$, it was decided to enshrine that measurement as the length of metal rod. Similarly, the original concept of the kilogram as the mass of a cubic decimetre of water was embodied as the mass of a platinum cylinder. These two artefacts, along with the second as 1/86 400 of a day, were the basis of the metric system of France. Other countries adopted this system, and an international Convention $d u$ Metre, signed on 20 May (now World Metrology Day) in 1875, established an International Bureau of Weights and Measures (the BIPM) to maintain, among other things, new artefacts: the International Prototypes of the Metre and of the Kilogram. The metre artefact remained the definition of length until $1960^{2}$, while the International Prototype of the Kilogram (IPK) will be the mass definition until 20 May 2019.

That well into the twenty-first century the definition of the kilogram is a nineteenth-century artefact based on an eighteenth-century object is more than just an embarrassment - it is scandalous. Since their manufacture late in the 1800s, the masses of the IPK and its presumptively identical copies have been diverging ${ }^{3}$, with some discrepancies larger than a part in $10^{7}$. Apparently, the masses of the IPK and/or its copies are drifting. But, by definition, the IPK is always one kilogram - if it acquires surface dirt, the rest of the

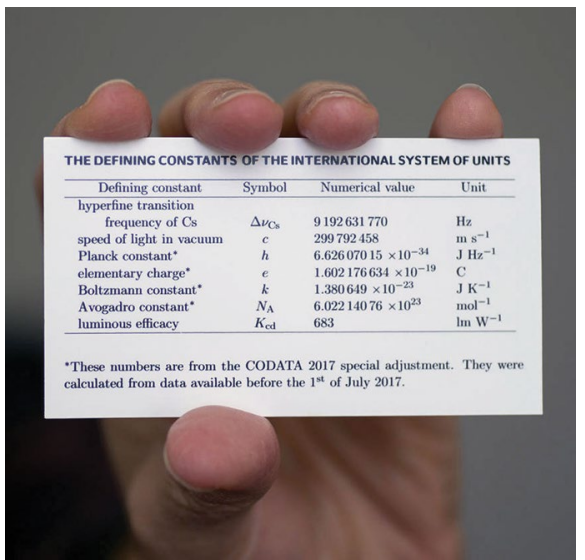

Credit: Stoughton / NIST

Universe loses mass. Fixing this scandalous situation was a major motivation for the current reform of the SI.

That reform follows the spirit of the final redefinition of the metre in $1983^{2,4}$. Soon after the 1960 replacement of the artefact definition with one based on the wavelength of light from a krypton lamp, metrologists found that laser light was a far better choice, and used it as a de facto standard. Another redefinition was needed, and the obvious choice would have been to adopt the de facto laser standard. That choice, however, would have invited further redefinition when a better light source appeared. Instead, metrologists made a beautiful and brilliant choice, defining the speed of light $c$ to be exact, so that the metre is defined as the distance that light travels in a certain time. That definition of the metre depended on the emerging technology of optical frequency measurement, and as both the quality of lasers and the ability to measure their frequency improves ${ }^{5,6}$, so does the definition of the metre.

The 2019 reform of the SI brings that beautiful concept of defining a unit by fixing the value of a constant of nature to four more SI units (pictured). Now Planck's constant $h$ defines the kilogram: recalling that the energy of a photon of frequency $\nu$ is $E=h \nu$, and the mass $m$ lost by an object emitting such a photon is $m=E / c^{2}=h \nu / c^{2}$, we see how defining $h$ and $c$ allows us to define mass. But 'weighing' photons is not accurate enough to replace the artefact kilogram. Instead, measuring forces on current-carrying wires (Kibble balance approach) and counting atoms in a perfect crystal (silicon sphere approach) both use the fixed value of $h$ to define the kilogram to a few parts in $10^{8}$, ending the reign of an artefact with a drifting mass.

Defining $h$ invites another, similarly beautiful and revolutionary idea: defining $e$, the electron charge. Not only does this replace the old definition of the ampere as the current that produces a certain force in a certain geometry, it provides fixed SI values for the Josephson constant $2 e / h$ and the von Klitzing constant $h / e^{2}$. Formerly, chosen values of these constants were used to provide the standards of 'conventional' (not SI) quantum measurements of voltage and resistance. Now, these quantum electrical standards are formally within the SI after decades of being separate.

With the new SI, we are indeed close to realizing the dream of units that are "for all times, for all people".

\section{William D. Phillips}

Joint Quantum Institute, University of Maryland and National Institute of Standards and Technology, Gaithersburg, MD, USA.

e-mail: william.phillips@nist.gov

Published online: 2 May 2019

https://doi.org/10.1038/s41567-019-0514-8

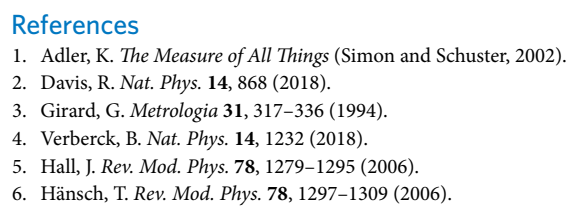

References

hings (Simon and Schuster, 2002).

Davis, R. Nat. Phys. 14, 868 (2018).

Verberck, B. Nat Phys, 14, 1232 (2018).

6. Hänsch, T. Rev. Mod. Phys. 78, 1297-1309 (2006)

\begin{tabular}{|c|c|c|c|c|c|c|c|c|c|c|c|c|c|c|c|c|}
\hline $\mathrm{m}$ & $e$ & $\stackrel{\AA}{A}$ & $\mathrm{~S}$ & $\mathrm{u}$ & $R$ & $E_{\mathrm{h}}$ & $F$ & $\Omega$ & ${ }^{\circ} \mathrm{R}$ & $\mu_{0}$ & $\varepsilon_{0}$ & $\alpha$ & $\sigma$ & V & $\mathrm{R}$ & $\mathrm{e}$ \\
\hline
\end{tabular}

